\title{
Propriété privée, équilibre social et organisation de l'espace
}

«Un fait capital domine toute la civilisation moderne, le fait que la propriété d'un seul peut s'accroître indéfiniment, et même, en vertu du consentement presque universel, embrasser le monde entier" (RECLUS 1908)

La description des structures agraires, chère à la géographie rurale traditionnelle, attribue une place de choix au thème de la propriété. La plupart des auteurs de thèses régionales $y$ ont consacré un chapitre, attentif qu'ils étaient à saisir les spécificités culturelles, économiques et sociales. Un sol fertile peut, par exemple, déterminer l'opiniâtreté de l'attachement à une terre et sa parcellisation excessive. Le phénomène s'inscrit dans le temps et résulte d'une longue évolution qui relève, comme l'écrivait joliment Jules Sion, des «influences les plus subtiles, les moins saisissables de l'histoire sociale et de la psychologie collective» (SION 1908). Ainsi reconstitué, l'état de la propriété semble figé et immuable tout comme la problématique. On s'intéresse à l'organisation actuelle de l'espace rural et à un paysan normalisé plutôt qu'à de petits exploitants ou de gros propriétaires, socialement contrastés. En effet, la dynamique sociale demeure souvent étrangère à ce type d'études aptes à dégager les harmonies des relations homme/milieu mais sourdes aux conflits qui sous-tendent la production des structures spatiales. Après 1950, la perspective se modifie et les thèses récentes situent l'évolution des structures foncières au cœur des mutations du monde contemporain, que ce soit en étudiant les réformes agraires du Tiers-Monde ou les transformations du paysage humanisé en Europe occidentale. La propriété privée du sol devient alors un trait culturel fondamental du monde européen, le principe de nonappropriation caractérisant les formations sociales précoloniales des autres continents (LACOSTE 1984), ce que nuancent néanmoins les anthropologues (WERDER 1978).

Dans l'analyse de la ville, la prise en compte des problèmes fonciers est l'un des apanages de la géographie "critique» à tendance marxienne. Cette dernière réactualise le constat, dressé au début de ce siècle par E. Reclus (l'un des inventeurs de la géographie sociale), et réinterprète la production de l'espace et la transformation du paysage comme manifestations de l'accumulation du capital (CORDEY 1978). Ce genre d'approche rejette les modèles des économistes lesquels privilégient l'étude des prix du sol en fonction des lois du marché. Une seconde variante qui ne cesse pourtant d'inspirer nombre de travaux de qualité (BOEHM 1980).
Reste que d'une manière générale, il manque encore à la géographie la théorie spatiale de l'appropriation que W.A. Gallusser appelle de ses vœux (GALlusser 1979a). Dans une telle entreprise, le rapport de la propriété du sol à son utilisation, l'analyse des structures foncières, la modélisation des systèmes qu'elle inspire, reviennent à prendre en compte la dimension sociale des espaces humanisés. Celle-ci relève de conditions matérielles mais aussi de codes et de valeurs. C'est dire qu'il importe de situer la place de la propriété dans le façonnement des «imaginaires sociaux». Par cette expression, Bronislaw Baczko désigne les «idées-images de la société globale et de tout ce qui se rapporte à elle» (BACZKO 1984). Les représentations mentales servent à structurer la pratique sociale, soit également à maîtriser l'espace au plan symbolique, en tissant un réseau de significations commun. Dès lors, non seulement les transferts de propriété seront partie intégrante de la dynamique spatiale (GALLUSSER 1979b), mais la perception même de cette réalité formalisée dans les règles du droit et vécue quotidiennement. Pour faire ressortir les virtualités de la problématique, il faut l'inscrire dans une «histoire des pratiques spatiales» (WALTER 1985a). Seule une géographie attentive à ne pas négliger la dimension temporelle peut saisir la subtilité des rapports que la société occidentale entretient avec la propriété privée depuis deux siècles. Cette prétention rejoint le souci de ceux qui ont mis comme condition à un projet géographique reformulé l'objectif de construire "un modèle de la réalité géohistorique de son tempS» (FERRIER, RACINE, RAFFESTIN 1978).

\section{Le paradigme individualiste}

Le rôle attribué à la propriété privée s'insère dans la structure analytique de type néo-classique qui veut que la croissance économique du monde occidental,

François Walter, Dr, Institut de Géographie, Université de Fribourg, Pérolles, 1700 Fribourg 
dont la révolution industrielle est un avatar, s'explique d'abord par l'émergence d'institutions favorables à l'initiative individuelle (NORTH, THOMAS 1970). La propriété privée est la première de ces institutions. Son existence justifie l'entreprise productive, individuellement et socialement. Le philosophe anglais du XVIIe siècle, John Locke, a formulé la théorie de l'individualisme possessif sur laquelle fonder aussi bien l'accumulation de richesses que le travail salarié (MACPHERSON 1971). Toutefois, c'est après 1750 que de tels principes vont acquérir toute leur force opératoire, en poussant au remaniement de la vieille conception féodale du droit de propriété et en servant l'avènement de la société bourgeoise.

En effet, l'ancienne coutume juridique distinguait «domaine direct» et "domaine utile». Le paysan ne disposait pas de la propriété entière du sol qu'il cultivait puisque le seigneur féodal conservait le domaine direct (autrement dit la propriété du sol en tant que telle). Seul l'usage de la terre lui était attribué. Dans la pratique, la confusion des deux types de propriété semble s'opérer au moment où le paysan obtient la reconnaissance de l'hérédité de la tenure et de son aliénabilité (déjà aux XII-XIIIe siècles). L'évolution postérieure amène à considérer de plus en plus la propriété utile comme la véritable propriété. Néanmoins, formellement, ce qu'on appelle en termes juridiques la "consolidation», c'est-à-dire la réunion sur un même bénéficiaire de droits jusqu'alors séparés, est un acquis des Lumières et de la Révolution française.

La notion de propriété individuelle, "droit inviolable et sacré» selon la formule célébrée à l'article 17 de la Déclaration des droits de l'homme et du citoyen (1789), inspire ensuite une codification formelle. En Suisse, on en retrouve les principes, mutatis mutandis, dans les Codes civils ainsi que dans toutes les constitutions cantonales du XIXe siècle (sauf celle du Tessin qui omet curieusement de garantir la propriété privée). Le vieux droit germanique subsiste uniquement sous la forme de traits considérés comme archaïques: la propriété collective (Allmend et corporation d'alpage) si importante dans le système helvétique des bourgeoisies. L'uniformisation des règles de possession de l'espace justifie également des entreprises gigantesques de cadastration: au début du XXe siècle, seuls sept cantons ont terminé la mensuration de leur territoire, parcelle par parcelle, en vue de dresser un plan et un état détaillé de la propriété. Le cadastre fournit les éléments nécessaires à un inventaire des ressources (la terre comme outil de production et base du système fiscal) tout en assurant la sécurité juridique de la propriété désormais contrôlée par l'Etat.

Fonder ainsi la vie sociale sur la propriété équivaut à promouvoir l'individu, bénéficiaire des nouvelles libertés coextensives à la propriété. La liberté économique ouvre toute grande la porte à la convoitise foncière - l'accès à la propriété ne focalise-t-il pas les stratégies paysannes? - mais aussi à l'exploitation du territoire et des ressources naturelles. «Le territoire de la France dans toute son étendue est libre comme les personnes qui l'habitent', proclame une loi rurale de 1794. En Prusse, le droit civil de 1794 accorde au propriétaire la liberté de construire comme bon lui semble sur sa parcelle. En Suisse aussi, les interdictions de bâtir en dehors du périmètre villageois pour préserver l'intégrité des surfaces cultivables sont levées. Le cas de Wohlen le démontre de manière spectaculaire: au XVIIIe siècle, la population avait doublé en s'entassant dans un nombre de maisons quasi inchangé. Elle double pratiquement de nouveau entre 1798 et 1850, mais, durant cette période, le nombre de bâtiments passe de 92 à 218 (DUBLER, SIEGRIST 1975)!

Plus généralement, c'est le libéralisme qui, en s'épanouissant, transforme les structures économiques. Le remplacement du «marché régulé par des marchés autorégulateurs» (POLANYI 1983) a des implications considérables sur la relation à l'espace. Primordialement parce que le libéralisme peut être considéré comme a-spatial: il se distancie des espaces concrets de la souveraineté politico-juridique pour dynamiser l'espace «abstraib) du marché sans délimitation absolue. Les recherches empiriques n'ont pas manqué de signaler combien, dès le XIXe siècle, l'activité industrielle détermine un nouveau rapport à la propriété. $\mathrm{R}$. Braun, par exemple, nous y rend attentif en étudiant les campagnes zurichoises (BRAUN 1965).

\section{Les répercussions spatiales}

Pourtant, le triomphe de l'individualisme et la généralisation progressive des rapports marchands, bien que basés sur des principes a-spatiaux a priori, ont pour conséquence une nouvelle traduction spatiale des structures économiques et sociales. En effet, les mécanismes économiques du capitalisme hiérarchisent spontanément l'espace et rendent de ce fait plus complexe l'analyse des régularités. L'ordre que l'on observe dans l'organisation de l'espace urbain au niveau du tracé viaire peut certes être mis en relation avec les limites du parcellaire. Sa fixité, formalisée dans les plans cadastraux, apparaît comme une constante dont les banlieues du siècle passé portent aujourd'hui encore la marque. Le quadrillage géométrique d'un quartier résidentiel ne provient pas, quant à lui, nécessairement ou mécaniquement d'une intervention planifiée des pouvoirs publics. C'est donc que la confiance du XIXe siècle dans l'efficacité du marché autorégulateur, loin d'être seulement une joyeuse utopie, se vérifie concrètement dans le paysage. Le libre jeu des lois du marché ordonne la ville selon un zoning spontané, qu'on a expliqué par généralisation empirique sous forme de différentes règles (gradient des localisations commerciales, gra- 
dient des densités, croissance allométrique, etc.). En simplifiant, la situation de chaque consommateur d'espace dépend d'une relation établie pour chaque utilisateur entre les désutilités liées aux transports et les utilités liées à l'emploi de la localisation. Tout cela se traduit en termes de prix, parce que le sol, transformé par l'appropriation est devenu une marchandise. On sait combien la suppression des entraves mises aux échanges immobiliers a été l'une des revendications fréquentes du processus d'abolition de la féodalité. Voilà pourquoi la nouvelle société libérale s'est attachée à concrétiser la liberté du commerce de la terre. Une fois le sol devenu marchandise, le marché foncier joue comme régulateur de l'allocation des terrains. Au XIXe siècle, la spéculation foncière, qui laisse les pouvoirs publics largement démunis, est essentielle à la compréhension de la structuration urbaine. B. Fritzsche l'a montré, de manière nuancée, pour le cas bernois (FRITZSCHE 1977).

A d'autres échelles, individualisme et économie de marché génèrent des formes d'organisation spatiale nouvelles. Les géographes ruralistes ont été attentifs à décrire les transformations du paysage aux XVIII-XIXe siècles sous l'effet de l'individualisme agraire triomphant. La liberté d'enclore, par exemple, entraîne la réification de l'appropriation lorsque les limites des propriétés sont soulignées par des haies, des clôtures et le bornage. Au-delà de ce phénomène, il y a, à l'échelle régionale et par l'effet spontané des lois du marché, la substitution d'une nouvelle organisation de l'espace à l'ancienne de type étroitement territorialisé. Manifestant une indépendance décisionnelle très grande, la nouvelle structure fait éclater le cadre régional existant et le désarticule. Ainsi, l'irruption de décisions externes par rapport au réseau informatif traditionnel déstabilise le système économique et social. Les producteurs de céréales d'Europe occidentale en ont fait l'expérience lorsque l'approvisionnement s'est greffé, après 1850, sur d'autres circuits économiques extérieurs au monde européen. N'est-ce pas là une manière de réaffirmer l'importance de la division territoriale du travail dans la genèse des disparités régionales?

\section{Le jeu délicat des restrictions}

Le principe du marché autorégulateur cède progressivement la place à une configuration nouvelle. L'Etat $y$ joue un rôle de plus en plus prépondérant en s'insérant dans le système économique et social. La règle selon laquelle la propriété privée est inviolable se trouve peu à peu corrigée. Si le dernier quart du XIXe siècle marque la fin du libéralisme intégral, il faut attendre l'après-seconde Guerre mondiale pour voir s'épanouir les tendances planificatrices. Parallèlement, mais avec des rythmes qui lui sont propres, la légitimité de la propriété privée est battue en brèche.
Une telle remise en cause du code possessif n'est rien d'autre qu'une forme nouvelle de perception des fonctions de la propriété privée dans le système économique et social. Cette révision affecte les pratiques spatiales par le biais des politiques d'urbanisme et d'aménagement.

Les juristes se sont montrés très attentifs à suivre les vicissitudes du droit de propriété depuis 1800 (STEINAUER 1981), unanimes à constater surtout l'accroissement des restrictions, voire à les interpréter comme un processus de "socialisation à froid» (GRISEL 1955). Pour nuancer leur discours, disons d'abord que les principes de l'individualisme possessif n'ont jamais été remis fondamentalement en cause et que la plus grande prudence a toujours été observée lorsqu'il s'agissait d'attenter à la propriété. L'expropriation pour cause d'utilité publique est, au XIXe siècle, un outil essentiel, rivé à la garantie de la propriété, dès sa genèse, comme le prouve la formulation même de l'article 17 de la Déclaration de 1789. Elle entre en ligne de compte lors de la réalisation de routes, de canaux, de chemins de fer. Elle est étendue plus tard aux travaux d'installation pour la production et le transport d'électricité. L'expropriation sert aussi de base légale lors des opérations d'urbanisme: les transformations de Paris sous le Second Empire sont précédées d'une adaptation des dispositions de la loi de 1841. En Italie, les premières normes urbanistiques sont à chercher dans la loi sur l'expropriation de 1865 . La plupart des cantons suisses disposent avant 1900 d'une véritable loi sur le sujet. Certaines contiennent des dispositions applicables à la réalisation des plans d'extension communaux.

Mais il y a d'autres instruments opératoires dont les géographes devraient suivre l'inclusion dans la législation aménagiste, étudier les rapports de force qui les font adopter, mettre en évidence leurs effets sur l'allocation des sols. L'émergence de l'idée selon laquelle il faut faire payer les bénéficiaires des dépenses consenties par la collectivité pour équiper de nouveaux quartiers ou améliorer les dessertes existantes reste à étudier. En effet, sous l'Ancien Régime, le principe qu'une rectification de tracé s'opère au bénéfice de la collectivité s'accompagnait dans la pratique d'une participation des propriétaires au financement des indemnités. Cette règle coutumière semble s'être estompée au XVIIIe siècle et au début du XIXe avant d'être réactivée dans la législation. Tous les cantons suisses, cependant, n'ont pas introduit les contributions d'équipement (Mehrwerts- ou Erschließungsbeitrag) dans leurs lois d'expropriation ou dans celles de police des constructions. La décision de taxer plus ou moins fortement les propriétaires dépend de rapports de forces complexes et spécifiques propres à l'histoire de chaque canton ainsi que de l'idéologie des autorités en fonction au moment de l'élaboration juridique. Le cas de la loi fédérale sur l'expropriation de 1930 est exemplaire: en l'occurrence, la majorité du Parlement repousse la proposi- 
tion qui prévoyait la récupération de $50 \%$ de la plusvalue consécutive à l'exécution des travaux d'intérêt public (WALTER 1985b). Autre domaine également significatif des attitudes face à la propriété privée, celui de l'imposition des gains immobiliers, soit une méthode un peu différente que la précédente dans le même but. Certains cantons l'introduisent déjà au début du XXe siècle.

La conception récente des plans d'aménagement locaux et régionaux, telle qu'elle s'impose après 1950 en Suisse, marque une étape décisive dans les restrictions à la propriété privée. Les juristes ne s'y sont pas trompés qui pensent que le «droit de propriété est en quelque sorte émasculé» (BEGUIN 1947) par les atteintes qui lui sont portées. D'autres y voient le retour à un «Etat policien» (REICHLIN 1947). Les restrictions sont effectivement multiples: elles vont des interdictions classiques à des obligations de faire. Elles introduisent par exemple des charges nouvelles de nature fiscale en vue de désamorcer les tensions sur le marché immobilier. Ainsi, l'idée de prélever une contribution de plus-value consécutive non plus strictement à la réalisation de travaux par la collectivité mais plus généralement à toute mesure d'aménagement. Dans ce domaine, la Grande-Bretagne est allée très loin, avec des antécédants qui remontent jusqu'au XVIIe siècle, époque où l'on prélevait une taxe sur les améliorations de valeur des propriétés dues à l'élargissement des rues de Londres. Le «Town and Country Planning Acts de 1947 équivaut à une sorte de nationalisation de la valeur potentielle des terres puisque tout aménagement suppose non seulement autorisation de l'Etat mais encore rétrocession intégrale de la plus-value dont bénéficie le fonds concerné (CULLINGWORTH 1982). Le système aboutit à paralyser en quelque sorte le marché foncier si bien qu'on en revint par la suite à des solutions plus souples, l'alternance travaillistes/conservateurs découpant des périodes restrictives et d'autres qui le sont moins. En Suisse, Bâle-Ville a introduit dans sa loi de 1977 la taxation des plus-values. Au plan fédéral, le projet de loi sur l'aménagement du territoire de 1972 comprenait à la fois les contributions classiques d'équipement et un système très général de prélèvement de la plus-value consécutive aux mesures de planification. C'est l'une des raisons fondamentales pour laquelle les milieux d'extrême-droite, rejoints par les associations patronales et paysannes ainsi que par celles des arts et métiers ont lancé le référendum et ont réussi à faire capoter la loi en votation populaire. La loi actuelle entrée en vigueur en 1980 se garde de toucher aux questions brûlantes de la propriété foncière en prônant de manière nuancée l'établissement par le droit cantonal d'un régime de «compensations permettant de tenir compte équitablement des avantages et des inconvénients majeurs qui résultent de mesures d'aménagement». Débat hautement significatif s'il en est dans l'évolution de la relation à l'espace!

\section{L'aménagement comme technologie sociale}

La réticence viscérale à heurter les intérêts des propriétaires et l'attention délicate à prévoir des compensations financières appartiennent aux constantes de l'histoire sociale européenne. Cependant, depuis une vingtaine d'années, le débat sur la réforme du droit de propriété est à l'ordre du jour, ce dont témoigne l'abondante littérature juridique (LENDI 1976, MACHERET 1981, MOOR 1976, STEINAUER 1981). En effet, non content d'aggraver la portée des limites à la jouissance individuelle du sol, ce que le droit traditionnel interprète en termes de restrictions, l'aménagement suscite un bouleversement des conceptions de la propriété. Martin Lendi, l'un des meilleurs spécialistes de ces questions, écrit: "Aufgrund des Raumplanungsrechtes (Nutzungsplanungsrecht) und im besonderen aufgrund des Rahmennutzungsplanes erhält das Grundeigentum einen nach den inhaltlichen Aussagen der Rahmennutzungsordnung unterschiedlichen Inhalt bezüglich der Nutzungsmöglichkeiten» (LENDI 1976). Avec le zoning, appliqué en Allemagne dès la fin du XIXe siècle et diffusé ensuite à des rythmes très variables dans les instruments de planification territoriale, le propriétaire se voit attribuer un droit de propriété différencié selon la localisation de ses bien-fonds. Cette remise en cause du paradigme individualiste transforme le concept de sol-marchandise, espace abstrait et universel caractérisé par son prix. L'espace redevient concret et doit se gérer comme tel (MOOR 1976). La valeur d'usage prime à nouveau sur la valeur d'échange, comme ce fut le cas dans les sociétés pré-industrielles. Les inégalités de lieu discriminent à nouveau les propriétaires de terre un peu comme sous l'Ancien Régime.

L'analogie ne s'arrête pas là. Sous couvert d'une évolution de l'usage de l'espace dans un sens plus social, c'est en fait à un retour vers la vieille distinction qu'opérait l'Ancien Régime entre "dominium directum» et "dominium utile», soit une théorie de la propriété multiple sur le sol, évoquée au début de cet article. Si l'on exclut les solutions radicales des pays à économie planifiée (nationalisation et municipalisation du sol), l'idée de dissocier propriété et droit de construire fait lentement du chemin. On a imaginé de circonscrire dans l'espace la propriété privée: audessus d'un seuil délimité en hauteur, l'espace appartiendrait à la collectivité. Plus pragmatiquement, la dissociation aboutit en Suisse à la pratique du droit de superficie (droit de construire sur un terrain dont on est locataire), en France à celle du bail à construire (le preneur s'engage à édifier des constructions et à les entretenir sur le terrain du bailleur), en GrandeBretagne, aux Pays-Bas et dans les pays scandinaves à des systèmes étendus de location des sols.

Cela montre que même dans un système enclin à privilégier les solutions technocratiques, l'aménagement est devenu, selon l'expression de J.-P. Gaudin, 
une «technologie sociale» (GAUDIN 1985). Les politiques spatiales peuvent s'interpréter comme autant d'institutions favorables individuellement et socialement. Elles impliquent au fur et à mesure qu'elles se développent des formules nouvelles d'équilibre social, c'est-à-dire de parité entre les bénéfices escomptés par les individus et ceux attendus par la société dans son ensemble. En agissant sur l'espace, on agit en définitive sur la société. Or, l'action sur l'espace dépend des nuances subtiles selon lesquelles on perçoit la fonction de la propriété privée.

$\mathrm{Au}$ XIXe siècle, ce sont encore les propriétaires fonciers qui détiennent la majorité dans la plupart des municipalités élues des pays de démocratie libérale. Le lobby propriétaire aurait pu bloquer toutes les mesures planificatrices urbaines. S'il les a acceptées, c'est qu'il y trouvait son intérêt. Dans les grandes villes d'Allemagne, les propriétaires fonciers ont veillé à empêcher la concurrence du sol bon marché en essayant d'indexer la hausse des prix à la croissance urbaine, règlements et plans d'extension étant établis dans l'intérêt des détenteurs de terrain (BOEHM 1980). Le premier quart du XXe siècle a été étudié pour le cas français par J.-P. Gaudin (GAUDIN 1985). Les propriétaires s'y organisent en groupe de pression avec leurs associations spécifiques. Ils manifestent leur inquiétude devant la montée des prescriptions édiliaires. Le triomphe des idéologies mélinistes dès la fin du XIXe siècle, réactivées ensuite dans l'entre-deuXguerres et durant la période de Vichy sous la forme d'utopies régressives ruralisantes magnifiant le travail indépendant, la famille élargie, la petite exploitation agricole, expliquent la timidité des lois d'urbanisme: en tous les cas, on respecte l'intérêt des propriétaires.

En Suisse, durant les années 1930-1950, ce sont les milieux ruraux qui s'affirment comme les principaux protagonistes de l'aménagement, dans une optique de défense de la terre menacée par l'urbanisation et l'industrialisation. La grande industrie soucieuse de rationalisation se rallie aux mesures interventionnistes avec l'objectif de planifier la croissance économique et la création de nouveaux emplois. Par la suite, dans les années soixante, les plus farouches opposants à l'aménagement du territoire se recruteront dans les classes moyennes et la petite bourgeoisie paysanne. Leur action retarde assurément la promulgation des bases légales.

Le zoning, dont on sait l'importance dans l'histoire des instruments aménagistes, peut certes s'interpréter en première instance comme une atteinte à l'aspect sacré de la propriété. Mais fondamentalement, il s'agit de protéger les propriétaires fonciers contre toutes sortes de «nuisances». Le cas américain est sans doute le mieux documenté et le plus spectaculaire. Dans les villes des Etats-Unis, le zoning a été utilisé par les municipalités pour exclure les bas revenus, discriminer les familles avec enfants, voire dans un but avoué de ségrégation raciale. Dans une certaine mesure, le zoning serait au XXe siècle la contrepartie de ce que fut au XVIIIe siècle le mouvement des enclosures (NELSON 1977). La clôture voulait affirmer la prédominance des droits individuels du propriétaire. Le zoning réintroduit des droits collectifs, ceux pour un groupe d'être protégé contre l'invasion de catégories sociales défavorisées ou contre la proximité d'activités bruyantes et polluantes. Nous voici loin d'un zoning, simple correcteur des effets pervers du marché, tel que le prônent certains théoriciens!

L'hypothèse selon laquelle l'intervention sur l'espace procède avant tout de la définition d'un nouvel équilibre social nous paraît donc riche d'implications, à condition de dépasser une lecture juridique littérale et de s'interroger sur la place de la propriété dans les représentations mentales. Pour les géographes particulièrement, l'étude des rapports de propriété et de leur configuration spatio-temporelle pourrait constituer une approche féconde des structures sociales. En effet, s'il s'agit bien en fin de compte de définir un espace social, pourquoi ne pas prendre comme base d'analyse une codification immédiatement spatialisable telle que l'offre la propriété? La démarche mérite plus ample exploration car elle présente l'immense avantage par rapport aux théories purement économiques de l'espace social (la division territoriale du travail par exemple) d'englober les dimensions politiques, idéologiques et culturelles. En outre, elle résiste sans mal à toute l'évolution contemporaine (depuis deux siècles) qui réduit l'espace à un simple support (RAFFESTIN, BRESSO 1979). En effet, la réflexion théorique sur la propriété privée prend une importance croissante au fur et à mesure que se développe le mouvement de déterritorialisation. L'enracinement au territoire jadis vécu symboliquement est devenu pur objet de normalisation juridique. Un concept spatialisé dans un environnement social déspatialisant, tel pourrait donc être l'apport de cette recherche à une théorie spatiale toujours en construction.

\section{Zusammenfassung}

Das Thema des Privateigentums ist für das Verständnis räumlicher Praktiken von grundlegender Bedeutung. Speziell in der Sozialgeographie ist das individualistische Paradigma untrennbar mit jeglichem Theorisationsversuch verbunden. Die Rolle des Privateigentums darf daher nicht bloss unter dem strukturellen Aspekt (z. B. der Verteilung des Grundbesitzes) betrachtet werden, sondern muss auch als eine im Verhalten dem Raume gegenüber verborgene gesellschaftliche Versinnbildlichung angesehen werden. Zur Hervorhebung der Dynamik benutzt der Verfasser einen geo-historischen Ansatz. Er legt zunächst die räumlichen Auswirkungen des individualistischen Paradigmas dar. Danach werden die dem Privateigentum im Rahmen der Verstädterungs- 
und Raumplanungspolitik auferlegten Beschränkungen abgehandelt. Daraus wird ersichtlich, dass diese für die Grundeigentümer restriktive Politik nichts anderes ist als eine «soziale Technologie», welche die gesamte Gesellschaft prägt. Jegliche (aktive) Raumordnung hat ihren Ursprung in der gesellschaftlichen Versinnbildlichung des Eigentums und setzt ein Gleichgewicht zwischen privaten und allgemeinen Interessen voraus. Die Untersuchung der Besitzverhältnisse und deren raum-zeitliche Konfiguration kann daher zu einem neuartigen Verständnis der Sozialstruktur beitragen.

\section{Références}

BACZKO, B. (1984): Les imaginaires sociaux. Mémoires et espoirs collectifs, Paris.

BEGUIN, G. (1947): Questions juridiques concernant le plan d'aménagement national et régional. In: Revue de droit suisse, Heft 4, 349-431.

BOEHM, H. (1980): Bodenmobilität und Bodenpreisgefüge in ihrer Bedeutung für die Siedlungsentwicklung, Bonn.

BRAUN, R. (1965): Sozialer und kultureller Wandel in einem ländlichen Industriegebiet im 19. und 20. Jahrhundert, Erlenbach-Zürich.

CORDEY, P. (1978): De l'espace produit à l'espace vécu. In: Geographica Helvetica, Nr. 2, 87-92.

CULLINGWORTH, J.B. (1982): Town and Country Planning in Britain, London.

DUBLER, A.-M.; SIEGRIST, J.-J. (1975): Wohlen. Geschichte von Recht, Wirtschaft und Bevölkerung, Aarau.

FERRIER, J.-P.; RACINE, J.-B.; RAFFESTIN, C. (1978): Vers un paradigme critique: matériaux pour un projet géographique. In: L'Espace Géographique, No 4, 291-297.

FRITZSCHE, B. (1977): Grundstückpreise als Determinanten städtischer Strukturen: Bern im 19. Jahrhundert. In: Zeitschrift für Stadtgeschichte, Stadtsoziologie und Denkmalpflege, Nr. 4, 36-54

GALLUSSER, W.A. (1979a): Über die geographische Bedeutung des Grundeigentums. In: Geographica Helvetica, Nr. 4 $153-162$.
GALLUSSER, W.A. (1979b): Veränderungen im Grundeigentum als Ausdruck der Raumdynamik. In: Siedlungsgeographische Studien. Festschrift für Gabriele Schwarz, Berlin/ New-York, 465-482.

GAUDIN, J.-P. (1985): L'avenir en plan: technique et politique dans la prévision urbaine 1900-1930, Seyssel.

GRISEL, A. (1955): Les restrictions de droit public à la propriété privée. In: Schweizerisches Zentralblatt für Staatsund Gemeindeverwaltung, Nr. 5, 89-98 et 113-120.

LACOSTE, Y. (1984): Unité et diversité du Tiers-Monde. Des représentations planétaires aux stratégies sur le terrain, Paris.

LENDI, M. (1976): Planungsrecht und Eigentum. In: Zeitschrift für schweizerisches Recht, Heft 1, 1-224.

MACHERET, A. (1981): Droit et politique de la propriété foncière en Suisse. In: Recht als Prozess und Gefüge. Festschrift für Hans Huber, Bern, 403-415.

MACPHERSON, C.B. (1971): La théorie politique de l'individualisme possessif de Hobbes à Locke, Paris.

MOOR, P. (1976): Aménagement du territoire et propriété privée. In: Revue de droit suisse, Heft 2, 365-479.

NELSON, R.H. (1977): Zoning and Property Rights. An Analysis of the American System of Land-Use Regulation, Cambridge.

NORTH, D. C.; THOMAS, R.P. (1970): An Economic Theory of the Growth of the Western World. In: The Economic History Review, No. 1, 1-17.

POLANYI, K. (1983): La Grande Transformation. Aux origines politiques et économiques de notre temps, Paris.

RAFFESTIN, C.; BRESSO, M. (1979): Travail, espace, pouvoir, Lausanne.

RECLUS, E. (1908): L'Homme et la Terre, T. 6, Paris.

REICHLIN, P. (1947): Rechtsfragen der Landesplanung. In: Zeitschrift für schweizerisches Recht, Heft 4, 171-347.

SION, J. (1908): Les paysans de la Normandie orientale, Paris.

STEINAUER, P.-H. (1981): La proprièté privée aujourd'hui. In: Revue de droit suisse, Heft 2, 117-246.

WALTER, F. (1985a): La géographie historique, une histoire des pratiques spatiales? In: Cahiers de l'Institut de géographie de Fribourg, No 3, 13-18.

WALTER, F. (1985b): Fédéralisme et propriété privée 1930-1950. Les attitudes face à l'aménagement du territoire en temps de crise et de pleins pouvoirs. In: DISP, Nr. 82 , 21-27.

WERDER, M. (1978): Eigentum und Verfassungswandel. Eine Untersuchung der anthropologischen Bedingtheit der Eigentumsordnung und ihrer verfassungsrechtlichen Gewährleistung, Diessenhofen. 